\title{
Large-Signal Dynamic Behavior of Distributed- Feedback Lasers Including Lateral Effects
}

\author{
S. F. Yu, R. G. S. Plumb, L. M. Zhang, M. C. Nowell, and J. E. Carroll
}

\begin{abstract}
The large-signal behavior of DFB lasers is analyzed, including lateral as well as longitudinal variations in carrier density, photon density, and refractive index. The effective index method and other approximations are used to reduce the complex three-dimensional problem to one dimension. The coupled wave and carrier rate equations are then solved in a self-consistent manner. Lateral spatial carrier hole burning and lateral diffusion are found to affect the relaxation oscillation frequency and damping rate of DFB lasers, depending on their detailed structure. The effective time-averaged linewidth enhancement factor is also affected.

In symmetric AR-coated $\lambda / 4$ phase-shifted lasers the side mode suppression ratio can be deteriorated significantly by lateral spatial hole burning when $k L$ is large.
\end{abstract}

\section{INTRODUCTION}

$\mathrm{E}$ XTENSIVE studies have concentrated on the largeand small-signal dynamic responses of FabryPerot(FP) lasers under the effect of lateral spatial hole burning (SHB) and carrier diffusion [1-6]. The dynamic responses of the lasers are strongly determined by their physical structure. For FP lasers with narrow stripes, strong carrier confinement, and index guiding, the lateral spatial hole burning of electron density in the active region causes gain compression and as a result, significantly damped relaxation oscillation can occur [4], [6]. The lateral carrier diffusion determines the strength of the damping of relaxation oscillation caused by SHB. The lateral carrier diffusion also varies the relaxation oscillation frequencies [2] and the dynamic wavelength shift (frequency chirp) [2], [3] of the devices. It has been shown that with optimized laser parameters, the small-signal (AM and FM) modulation bandwidth can be increased by several gigahertz [2] and the frequency chirp can be reduced by several gigahertz [2]. In FP lasers, the longitudinal variation of carrier density is small and the lateral SHB and carrier diffusion effect is fairly constant along the longitudinal direction of the laser. This implies that the lateral effect is more significant than longitudinal effects in FP lasers.

On the other hand, the dynamic and static characteristics of distributed-feedback (DFB) lasers are affected by longitudinal SHB. The longitudinal carrier SHB induces gain compression [7] and the nonuniform index profile

Manuscript received May 10, 1993; revised November 29, 1993. The work of S. F. Yu was supported in part by the Croucher Foundation of Hong Kong and by the Universities Funding Council under an O.R.S. Award.

The authors are with the Department of Engineering, Cambridge University, Cambridge CB2 1PZ, England.

IEEE Log Number 9402332. varies the modulation response of the lasers [8]. In addition, for $\lambda / 4$ phase-shifted DFB lasers with large $\kappa L$, the large number of photons accumulated around the phaseshifted region causes severe hole burning and this reduces the side mode suppression ration (SMSR) of the lasers.

However, for low $\kappa L$ devices, longitudinal SHB is lower and the lateral effect may be significant in their dynamic behavior. Inaccurate estimation of the dynamic behavior of DFB lasers may result if we ignore the importance of lateral effects. For strong longitudinal SHB, the lateral diffusion effect is no longer uniform along the laser cavity and lateral carrier diffusion may modify the effective longitudinal carrier profile of the laser, and hence, the SMSR.

In this paper, we compare and analyze two-dimensional SHB effects on the dynamic behavior of DFB lasers, including the consideration of different laser structures. We also analyze the effect of lateral SHB and carrier diffusion on mode profiles in the longitudinal direction. Detailed three-dimensional laser structures are considered in our analysis. The three-dimensional problem is simply converted into one-dimensional coupled-wave equations through the effective index method [9] and the perturbation method [10]. In order to reduce the excessive computing time in computing the lateral SHB, first-order approximation of lateral carrier distribution [2], [5] is employed in our model. Using this approximation, the two-dimensional variation of the refractive index can be taken into account; a higher order approximation could be used in the same way. The dynamic behaviors of the lasers can then be solved self-consistently by using the corresponding time-dependent coupled-wave equations and rate equations of carrier concentration.

The paper is organized as follows: in Section II we present a simple self-consistent dynamic model with the lateral as well as longitudinal SHB taken into account. In Section III we present the numerical simulation results: 1) comparison between the lateral and longitudinal SHB effect on the dynamic behavior of the lasers, 2) lateral effect on the longitudinal modes, and 3) the lateral effect on the effective time-averaged linewidth enhancement factor under large-signal modulation. A brief discussion and conclusion will be given in Section IV.

\section{Dynamic Model}

\section{A. Laser Structure for the Model}

A typical structure of $1.55 \mu \mathrm{m} \mathrm{BH} \mathrm{InGaAsP} \mathrm{DFB} \mathrm{laser}$ with second-order gratings is shown in Fig. 1. Although 
second-order gratings are considered in our analysis, first-order gratings can also be studied by neglecting the radiation loss coefficient in the coupled-wave equations. Fig. 1(a) shows the side view of the laser. We assume that the structure of the laser is composed of five layers of semiconductor materials: the InP cladding layer, the InGaAsP $(1.3 \mu \mathrm{m})$ guiding layer, the InGaAsP $(1.55 \mu \mathrm{m})$ active layer, the InGaAsP $(1.3 \mu \mathrm{m})$ buffer layer, and the InP substrate. The refractive indexes of the materials in these layers are $n_{c}, n_{g}, n_{a}, n_{b}$, and $n_{s}$, respectively. The refractive indexes of the cladding and substrate layers are assumed to be the same. The grating is formed on the guiding layer and has a trapezoidal shape with an angle of $54^{\circ}$. The end view of the laser is shown schematically in Fig. 1(b). The InGaAsP guiding layer, active layer, and buffer layer are surrounded by the InP on all sides. The change in the dielectric constant between the InP and InGaAsP material confines the photons in the lateral and transverse directions. In the following calculations, only the fundamental-transverse modes and lateral modes will be considered.

\section{B. Theory}

From Maxwell's equations, we can write the time-dependent wave equation as

$$
\nabla^{2} E=\frac{1}{c^{2}} \frac{\partial^{2} \epsilon E}{\partial t^{2}}
$$

where $x, y$, and $z$ are the transverse, lateral, and longitudinal directions of the waveguide and $\epsilon$ is the complex permittivity in the waveguide. The transverse electric (TE) modes in the waveguide can be approximated as

$$
E(x, y, z, t)=\phi(x) \psi(y) E(z, t) e^{-j \omega_{0} t}
$$

where $E(z, t)$ is the slowly varying longitudinal field amplitude. The normalized transverse and lateral field profiles $\phi$ and $\psi$ are assumed time independent. From the Maxwell's equation (1), a set of coupled-wave equations for a resonant situation can be derived by combining the perturbation solution of the Floquet-Bloch expansion theory [10] and the conventional coupled-mode equations for the two guided waves. For a second-order grating, we take the longitudinal propagating field within the laser $E_{0}$ and the field within the laser leading to surface radiation $E_{-1}$ as

$E_{0}(x, y, z, t)=\phi_{0}(x) \psi_{0}(y)\left(R(z, t) e^{-j \beta_{0} z}+S(z, t) e^{j \beta_{0} z}\right)$

$E_{-1}(x, y, z, t)=\phi_{-1}(x) \psi_{-1}(y)[R(z, t)+S(z, t)]$

where $R$ and $S$ are the slowly varying longitudinal forward and reverse propagating field amplitudes and $\beta_{0}$ is the propagation constant at Bragg frequency. The effective index method [9] is used to reduce the two-dimensional wave equation to one dimension in the $z$ direction. Fig. 2 shows the application of effective index method in solving the two-dimensional problem in a one-dimensional treatment. We assume the field profiles in the $x$ and $y$

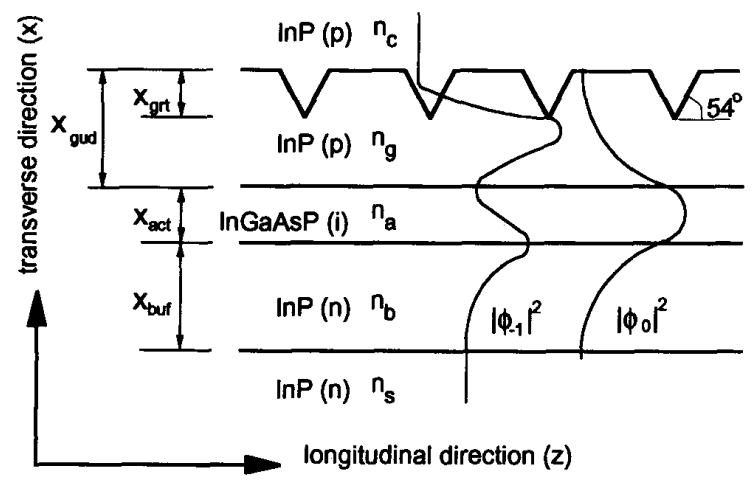

(a)

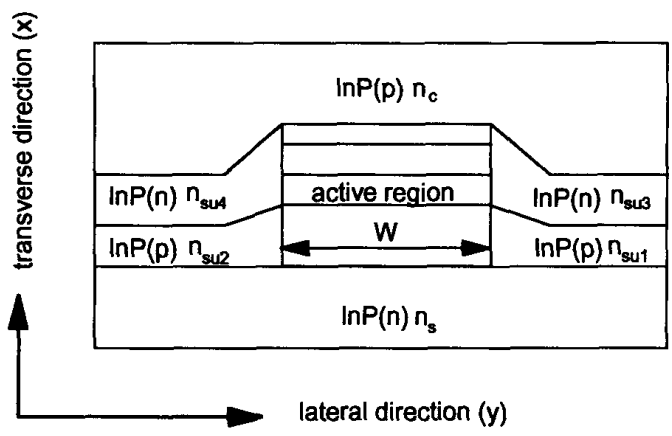

(b)

Fig. 1. (a) Schematic side view of the DFB lasers investigated in this paper. $X_{\text {grt }}, X_{\text {gud }}, X_{\text {act }}$, and $X_{\text {buf }}$ represent the grating depth and the thickness of the guiding layer, active layer, and buffer layer, respectively. The transverse intensity profiles of the guided mode and the radiation mode $\phi_{0}$ and $\phi_{-1}$ are also shown. (b) The cross section of the DFB lasers. $n_{\mathrm{su} 1}, n_{\mathrm{su} 2}, n_{\mathrm{su} 3}$, and $n_{\mathrm{su} 4}$ represent the refractive indexes surrounding the waveguide in the lateral directions. $w$ is the width of the active layer.

directions satisfy

$$
\begin{gathered}
{\left[\frac{\partial^{2}}{\partial x^{2}}+n_{0}^{2}(x, y) k_{0}^{2}-\beta_{\text {eff }}^{2}(y)\right] \phi(x)=0} \\
{\left[\frac{\partial^{2}}{\partial y^{2}}+\left(\beta_{\text {eff }}^{2}(y)-\beta^{2}\right)\right] \psi(y)=0}
\end{gathered}
$$

where $\beta_{\text {eff }}$ is the effective propagation coefficient in lateral direction and $\beta$ is the propagation constant to be determined. Caution should be used in calculating the propagation coefficient close to its cutoff value, as the effective index method becomes inaccurate [11]. From the above, we can obtain the time-dependent coupled-wave equations for fields $R$ and $S$, given as

$$
\frac{1}{\nu_{g}} \frac{\partial R}{\partial t}+\frac{\partial R}{\partial Z}=\left(g+i \delta \beta-\alpha_{s}-h_{1}\right) R+i\left(\kappa+i h_{1}\right) S
$$

$$
\begin{aligned}
-\frac{1}{\nu_{g}} \frac{\partial S}{\partial t}+\frac{\partial S}{\partial z}=-\left(g+i \delta \beta-\alpha_{s}\right. & \left.-h_{1}\right) S \\
& -i\left(\kappa+i h_{1}\right) R
\end{aligned}
$$




\begin{tabular}{|c|c|c|}
\hline$n_{c}$ & $n_{c}$ & $n_{c}$ \\
$n_{\text {su4 }}$ & $n_{g}$ & $n_{\text {su3 }}$ \\
\cline { 2 - 3 }$n_{\text {st2 }}$ & $n_{a}$ & $n_{\text {su1 }}$ \\
\cline { 2 - 3 } & $n_{b}$ & $n_{s}$ \\
\hline$n_{s}$ & $n_{s}$ & $n_{1}$ \\
\hline (I) & (II) & (III)
\end{tabular}

(a)

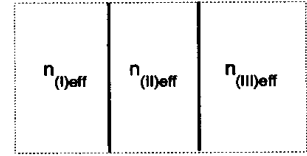

(b)
Fig. 2. The buried waveguide shown in Fig. 1(b) treated by the effective index method. (a) Three equivalent guides in the $y$ direction. (b) Resultant effective guide in the $x$ direction.

where $\alpha_{s}$ is the absorption and scattering loss in the waveguide and $g$ is the field gain given by

$$
g(z, t)=\frac{1}{2} \int_{\text {active }} \Gamma_{x} g_{N}\left(N(y, z, t)-N_{0}\right) \psi_{0}^{2}(y) d y
$$

where $N$ is the carrier concentration distribution in the lateral and longitudinal directions, $\Gamma_{x}$ is the transverse confinement factor, $g_{N}$ is the differential gain, $\nu_{g}$ is the group velocity, and $P$ is the photon density and is equal to the sum of the magnitude squared of the forward and reverse field amplitudes. $\kappa$ is the longitudinal coupling coefficient and $h_{1}$ is the first-order radiation loss coefficient. The expressions for $\kappa$ and $h_{1}$ are given in Appendix I.

The deviation from the Bragg condition $\delta \beta$ is given as

$$
\delta \beta=\frac{\omega_{0}}{c} n_{\mathrm{el}}(z, t)-\frac{\pi}{\Lambda}
$$

where $c$ is the speed of light and $\Lambda$ is the pitch of the grating. $n_{\mathrm{el}}$ is the effective refractive index in the longitudinal direction. The effective refractive index is related to the carrier density by

$$
n_{\mathrm{el}}=n_{\mathrm{el} 0}-\frac{\Gamma_{x} \alpha_{m} \gamma_{0}}{4 \pi} g_{N} \Delta N_{p}(z, t)
$$

where $n_{\mathrm{el} 0}$ is the refractive index at the carrier density $N=N_{0}, \alpha_{m}$ is the material linewidth enhancement factor, $\lambda_{0}$ is the free-space wavelength, and $\Delta N_{p}$ is the small change of the average carrier distribution in the longitudinal direction. Here, we assume that the longitudinal modes are affected only by the average refractive index distribution in the longitudinal direction; however, this average refractive index is modified by the lateral SHB.

The time-dependent rate equation of the carrier concentration is given by

$$
\begin{aligned}
\frac{d N(y, z, t)}{d t}= & \frac{J(y, z, t)}{q d}-\frac{N(y, z, t)}{\tau} \\
& -\nu_{g} g_{N}\left(N(y, z, t)-N_{0}\right) P(y, z, t) \\
& +D \nabla^{2} N(y, z, t)
\end{aligned}
$$

where $J$ is the injection current density profile, $d$ is the thickness of the active layer, $D$ is the diffusion constant, $\tau$ is the carrier lifetime, and $q$ is the electron charge. The photon density distribution $P$ is given as

$$
\begin{aligned}
P(y, z, t) & =\left[|R(z, t)|^{2}+|S(z, t)|^{2}\right] \psi_{0}^{2}(y) \\
& =P(z, t) \psi_{0}^{2}(y) .
\end{aligned}
$$

To simplify our calculation, the inhomogeneous carrier distribution in the lateral direction is expanded in a Fourier series. The first-order approximation of the carrier distribution is then given by [2], [5]

$$
N(y, z, t)=N_{p}(z, t)-N_{1}(z, t) \cos (2 \pi y / w)
$$

where $w$ is the width of the active region, $N_{p}$ is the average carrier distribution at a particular cross section, and $N_{1}$ is the perturbation of the carrier concentration in the lateral direction. Substituting (11) into (6) and (9), with $J$ assumed constant across the active layer and integrating (6) and (9) over the active layer, and including the gain compression coefficient $\epsilon$, we obtain the modified field gain expression given as

$$
g(z, t)=\frac{\Gamma_{x} g_{N}\left\{\Gamma_{y}\left(N_{p}(z, t)-N_{0}\right)-\xi_{1} N_{1}(z, t)\right\}}{2[1+\epsilon P(z, t)]}
$$

and the rate equations of the carrier densities given as

$$
\begin{aligned}
& \frac{d N_{p}(z, t)}{d t}= \frac{J(z, t)}{q d}-\frac{N_{p}(z, t)}{\tau} \\
&-\frac{\nu_{g} g_{N}\left\{\Gamma_{y}\left(N_{p}(z, t)-N_{0}\right)-\xi_{1} N_{1}(z, t)\right\}}{[1+\epsilon P(z, t)]} \\
& \cdot P(z, t)+D \frac{\partial^{2} N_{p}(z, t)}{\partial z^{2}} \\
& \frac{d N_{1}(z, t)}{d t}= \frac{2 \nu_{g} g_{N}\left\{\xi_{1}\left(N_{p}(z, t)-N_{0}\right)-\xi_{2} N_{1}(z, t)\right\}}{[1+\epsilon P(z, t)]} \\
& \cdot P(z, t)-\frac{1+\gamma}{\tau} N_{1}(z, t)+D \frac{\partial^{2} N_{1}(z, t)}{\partial z^{2}}
\end{aligned}
$$

Where $\Gamma_{y}$ is the confinement factor in the lateral direction and $\xi_{1}$ and $\xi_{2}$, respectively, are the first- and second-order coupling parameters of the carriers and the optical field $\psi_{0}$. Their definitions are given in Appendix I. The parameter $\gamma$ is given by

$$
\gamma=\left(\frac{2 \pi L_{\text {eff }}}{w}\right)^{2}
$$

where $L_{\text {eff }}$ is the effective diffusion length and is defined as $L_{\text {eff }}=\sqrt{D \tau}$.

The parameters given in (5) and (12) $\left(\kappa, \Gamma_{y}, \xi_{1}, \cdots,\right)$ vary slightly with the carrier-induced refractive index change in the active region. However, they are assumed to be constant with a value derived from the initial calculations. If we assume that the optical field has a sinusoidal profile and is well confined in the lateral direction, we get $\Gamma_{y}=$ $1.0, \xi_{1}=0.5$, and $\xi_{2}=0.5$. If the field penetrates outside the active region, these values are reduced. Table I shows 
the values of these parameters with different laser structures.

A self-consistent large-signal calculation can be obtained by solving (5) and (12) simultaneously. There exist several mathematical algorithms that can be employed to solve these equations such as the frequency domain power matrix method [12] and the time domain traveling wave method [13], [14]. In our calculation, the time domain method is employed to solve the large-signal dynamic behavior of the DFB lasers.

In our calculation, the longitudinal SHB is taken into account by dividing the laser longitudinally into a number of small equal sections. In each section, there is an average carrier concentration $N_{p}$ that is assumed to be constant in that section. There is also a perturbation of carrier concentration $N_{1}$ that arises from the lateral SHB. The values of $N_{p}$ and $N_{1}$ are allowed to change from section to section. The spontaneous noise coupled into the forward and reverse fields is introduced into (5). The detailed time domain calculation can be found in [14].

\section{Numerical RESULtS}

In the following calculation, the lengths of the lasers are $400 \mu \mathrm{m}$, and they are divided into 40 equal-length sections. A transfer matrix is introduced in each section to ensure the accuracy of the calculation. We ignore the longitudinal carrier diffusion as the separation between sections is much longer than the diffusion length. $\lambda / 4$ DFB lasers with first-order gratings and both facets perfectly antireflection coated are employed to investigate the dynamic behavior with lateral effects. The value of $h_{1}$ is set to zero in (5) and the effect of radiation loss is not considered. Nonlinear gain compression of value $\epsilon=1.0$ $\times 10^{-17} \mathrm{~cm}^{3}$ is introduced. The other parameters used in the calculation are given in Table II.

\section{A. Relaxation Oscillation Frequency and Damping Rate}

Fig. 3 shows the variation of the normalized relaxation oscillation strength and period with effective diffusion length. The relaxation oscillation strength is defined as the first overshoot power divided by the subsequent undershoot [1], [6]; the greater the oscillation strength, the less the damping will be. The lasers are biased just at threshold and modulated with a step function. The modulation amplitude is 2 to 2.5 times threshold and the steady-state optical power from the AR facets is around 8 $\mathrm{mW}$.

The structures under investigation are $\lambda / 4$ DFB lasers with $\kappa L=0.8,1.24$, and 3.2 . The value of the coupling coefficient is assumed to be changed by the height of the grating. The variation of $\Gamma_{x}$ is small in this range of grating depth and is assumed to be unchanged. The widths of the active region in $\mu \mathrm{m}$ are set to $w=3.0,2.5,1.5$, 1.25 , and 1.0 (types A-E, see Table I). For $\kappa L=1.24$, the longitudinal carrier distribution is relatively uniform. For $\kappa L=3.2$, SHB is strong in the phase-shift region, but for $\kappa L=0.8 \mathrm{SHB}$ is strong near the facets. Fig. 4 shows the longitudinal carrier distributions in lasers with $\kappa L=0.8$,
TABLE I

Parameters Defining the Lateral Structure of the Lasers

\begin{tabular}{|c|c|c|c|c|}
\hline Type & $w(\mu \mathrm{m})$ & $\Gamma_{x}$ & $\xi_{1}$ & $\xi_{2}$ \\
\hline A & $>3.0$ & 1.00 & 0.50 & 0.50 \\
\hline B & 2.50 & 0.99 & 0.40 & 0.48 \\
\hline C & 1.50 & 0.975 & 0.33 & 0.47 \\
\hline D & 1.25 & 0.96 & 0.30 & 0.46 \\
\hline E & 1.00 & 0.935 & 0.25 & 0.45 \\
\hline $\mathrm{F}^{*}$ & 1.50 & 0.97 & 0.3 & 0.465 \\
\hline $\mathrm{G}^{+}$ & 1.50 & 0.95 & 0.3 & 0.45 \\
\hline
\end{tabular}

This set of parameters represents the lasers, while $\kappa L$ varies between 0.8 and 3.2 (which corresponds to a change of the height of the grating $X_{\text {grt }}$ by $0.075 \mu \mathrm{m}$ ). These parameters do not change much in this range of $\kappa L$.

*This is the extreme case where the grating occupies the whole guide layer thickness, i.e., $X_{\text {gud }}=X_{\text {grt }}$.

${ }^{+}$These parameters are obtained only when the refractive indexes surrounding the active layers are set to 3.25 , as opposed to 3.17 used elsewhere.

TABLE II

PARAMETERS USED IN THE MODEL

\begin{tabular}{|c|c|}
\hline Carrier lifetime $(\tau)$ & $3 \times 10^{-9} \mathrm{~s}$ \\
\hline Differential gain $\left(\mathrm{g}_{\mathrm{N}}\right)$ & $3 \times 10^{-16} \mathrm{~cm}^{2}$ \\
\hline Transparency carrier density $\left(\mathrm{N}_{0}\right)$ & $1.5 \times 10^{18} \mathrm{~cm}^{-3}$ \\
\hline Linewidth enhancement factor $\left(\alpha_{m}\right)$ & 4.86 \\
\hline Absorption and scattering loss(energy) in waveguide $\left(\alpha_{s}\right)$ & $20 \mathrm{~cm}^{-1}$ \\
\hline Effective phase refractive index $\left(n_{\text {min }}\right)$ & 3.283 \\
\hline Effective group refractive index $(\mathrm{n})$ & 3.70 \\
\hline Length of the laser cavity $(\mathrm{L})$ & $400 \mu \mathrm{m}$ \\
\hline Thickness of the active layer (d) & $0.18 \mu \mathrm{m}$ \\
\hline Approximate emission wavelength $\left(\lambda_{0}\right)$ & $1.55 \mu \mathrm{m}$ \\
\hline Nonlinear gain suppression coefficient $(\varepsilon)$ & $1 \times 10^{-17} \mathrm{~cm}^{3}$ \\
\hline Refractive index of cladding layer $(n)$ & 3.17 \\
\hline Refractive index of guiding layer $\left(\mathrm{n}_{\mathrm{o}}\right)$ & 3.4 \\
\hline Refractive index of cladding layer $\left(\mathrm{n}_{\mathrm{n}}\right)$ & 3.54 \\
\hline Refractive index of cladding layer $\left(\mathrm{n}_{\mathrm{t}}\right)$ & 3.4 \\
\hline Refractive index of cladding layer $\left(n_{2}\right)$ & 3.17 \\
\hline Refractive index of sidewall $\left(n_{s w 1}, n_{s, 2}, n_{v 23}, n_{s, 4}\right)$ & 3.17 \\
\hline Thickness of guiding layer $\left(\mathrm{X}_{\text {aud }}\right)$ & $0.39 \mu \mathrm{m}$ \\
\hline Thickness of active layer $\left(\mathrm{X}_{\mathrm{ac}}\right)$ & $0.18 \mu \mathrm{m}$ \\
\hline Thickness of buffer layer $\left(\mathrm{X}_{\text {tuf }}\right)$ & $0.2 \mu \mathrm{m}$ \\
\hline Transverse confinement factor $\left(\Gamma_{x}\right)$ & 0.35 \\
\hline
\end{tabular}

1.24 , and 3.2 without the lateral effect. In our time domain model, the spontaneous emission noise is generated from a Gaussian-distributed random number generator [15]; this implies that the spontaneous emission coupled into the propagating fields is slightly different in each run of the calculation even with the same set of laser parameters. This varies the relaxation oscillation strength and period slightly. Fig. 3 shows average curves for several runs of the calculations.

The lateral and longitudinal effects on the relaxation oscillation strength and period can be summarized as below (note that other laser structures will show the same effects, but possibly with different relative magnitudes).

1) In the range of $\kappa L, 0.8$ to 3.2 , the longitudinal SHB has less of an effect on the dynamic response of the lasers than the lateral carrier diffusion and SHB. The reduction of oscillation strength can be as large as 90 percent or higher by the lateral effect. 


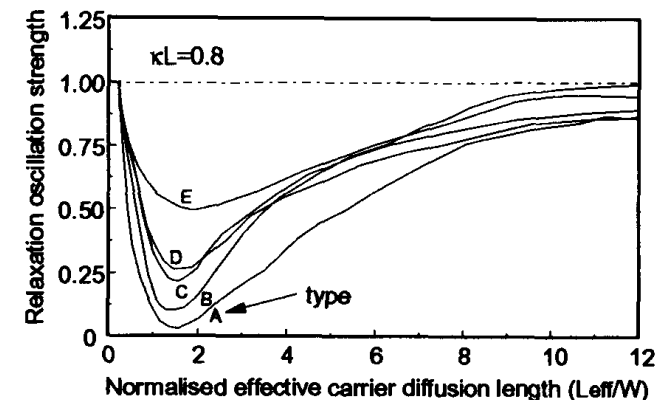

(a)

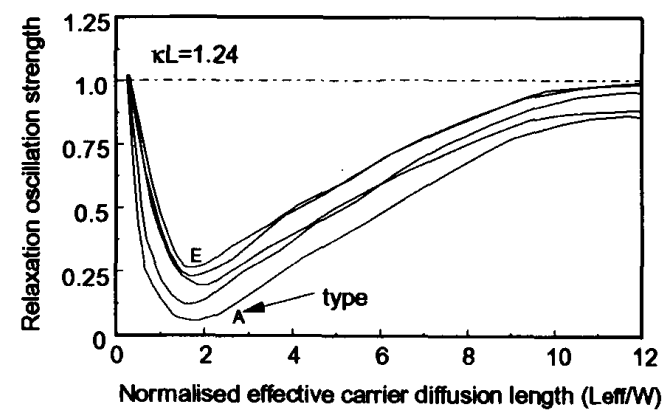

(c)

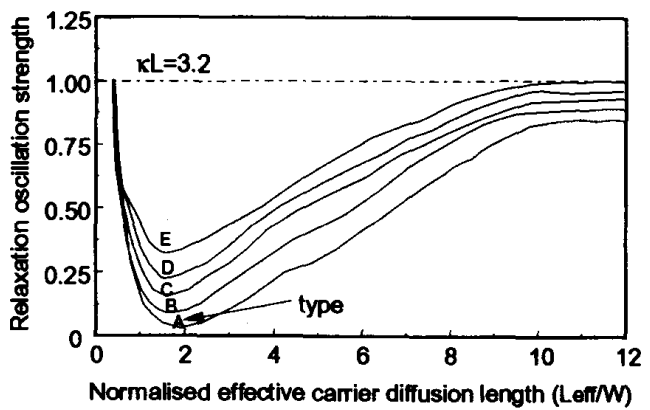

(e)

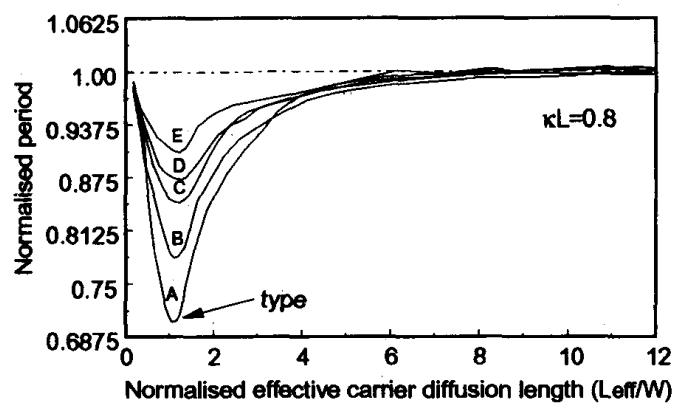

(b)

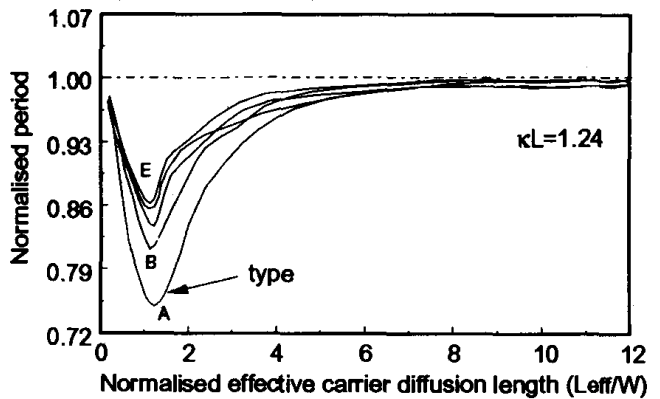

(d)

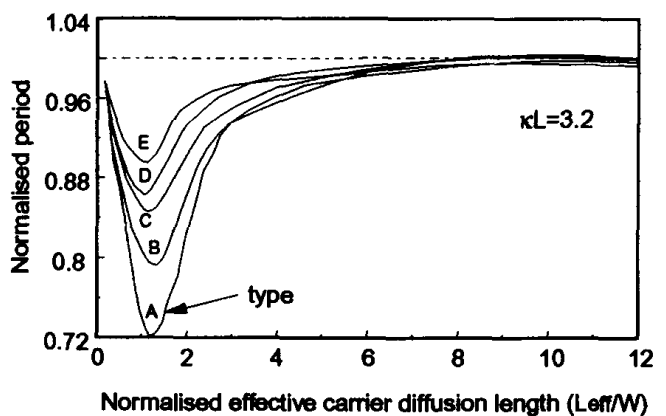

(f)

Fig. 3. Variation of the normalized relaxation oscillation strength and period with the normalized effective carrier diffusion length for lasers type A-E. (a) and (b) $\kappa L=0.8$; (c) and (d) $\kappa L=1.24$; (e) and (f) $\kappa L=3.2$.

2) The damping and the oscillation frequency are reduced as the width of the active region get smaller. This is because the lateral coupling between the optical field and the carrier concentration is reduced. In this range of $\kappa L$, lasers with type A structures have maximum damping and relaxation oscillation frequencies.

3) The relaxation oscillation damping and frequency vary with the normalized carrier diffusion length $\left(L_{\mathrm{eff}} / w\right)$; a value between 1 and 2 gives minimum oscillation strength and maximum frequency, and hence, may be optimum for high-frequency direct modulation. This optimum is relatively broad and depends only weakly on the width of the active region $w$, and the value of $\kappa L$. For large values of $L_{\text {eff }} / w(>10)$, the oscillation strength and period are close to the values calculated without considering the lateral effect; this is because the lateral carrier distribution is almost uniform. As $L_{\text {eff }} / w$ reduces to the order of unity, spatial changes in the carrier density become comparable with the active region width, and diffusion damping of relaxation oscillation becomes significant. Note that in this analysis, the carrier recombination time $\tau$ is not varied with $L_{\text {eff }}$, so reducing $L_{\text {eff }} / w$ corresponds to reducing the diffusion coefficient.) As $L_{\text {eff }} / w$ is reduced further still, strong lateral hole burning will still occur, but the movement of the carriers is over distances smaller than the active width, which has little effect, and diffusion damping becomes insignificant again, as for $L_{\text {eff }} / w$ large. Fig. 5(a) shows the dynamic response of a "type A" laser (see Table I) with $\kappa L=0.8$ with $L_{\text {eff }} / w$ varied from 5.0 down to 0.5 , and also the simpler case including no lateral effects.

The effects of the gain compression factor $\epsilon$ interact strongly with lateral diffusion. Values of $\epsilon$ from $1 \times 10^{-17}$ up to about $7 \times 10^{-17}$ are currently used in the literature, 


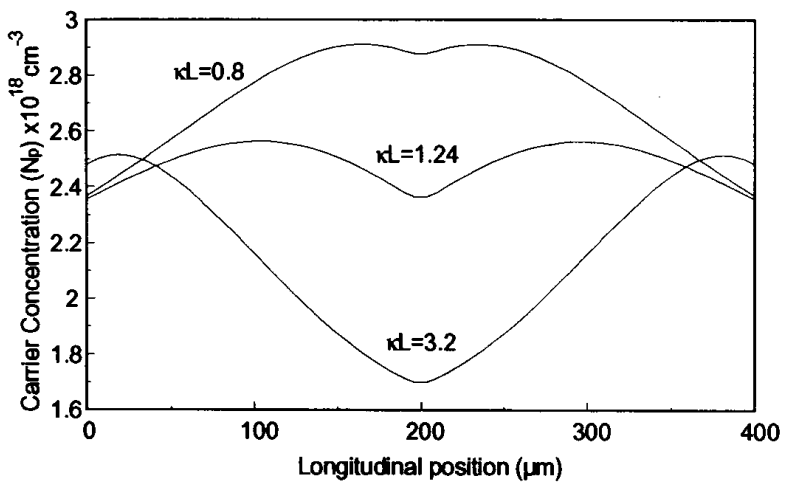

Fig. 4. The longitudinal average carrier concentration profile $N_{p}$ of the lasers with $\kappa L=0.8,1.24$, and 3.2 .

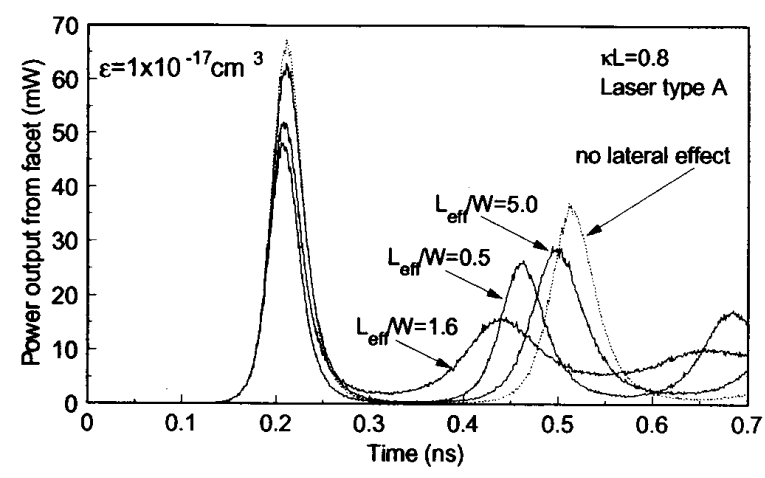

(a)

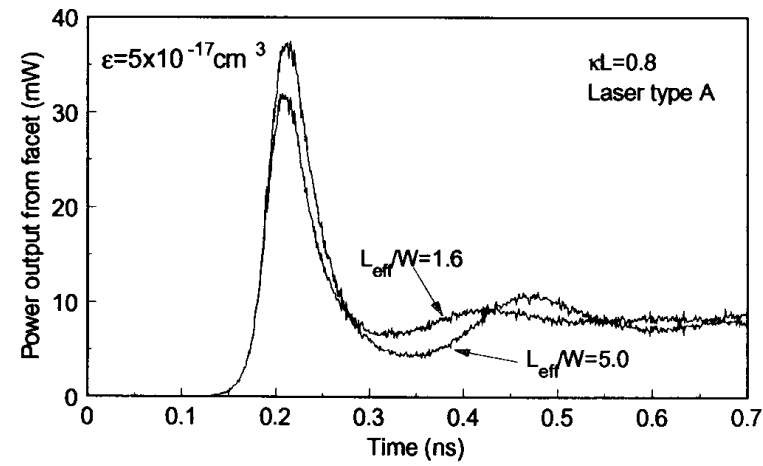

(b)

Fig. 5. Comparison between the transient response of power output from the facets with and without lateral effect. The effective carrier diffusion length is set to $L_{\mathrm{eff}} / w=1.6$ and 5.0. The laser has $\kappa L=0.8$ and is under large-signal modulation, (a) $\epsilon=1 \times 10^{-17} \mathrm{~cm}^{3}$ and (b)
$\epsilon=5 \times 10^{-17} \mathrm{~cm}^{3}$.

depending on the laser structure and matters such as carrier transport in quantum-well structures. Fig. 5(a) uses $1 \times 10^{-17}$ (at the low extreme of the range), which makes the effects of lateral diffusion very obvious. Fig. 5(b) uses $5 \times 10^{-17}$, which is toward the high end of accepted values and the effects of lateral diffusion are less, although still clearly visible. Lateral diffusion is seen to have a roughly comparable effect on laser response to direct gain compression, and since values for the latter are derived from observed laser responses, it may be that true values for gain compression are slightly lower than used previously, with lateral diffusion making up the difference.

The interaction between lateral and longitudinal carrier profiles is difficult to explain concisely, but may be described as follows. Fig. 6(a) shows the dynamic response of the average carrier density profile $N_{p}$. The lateral SHB strength profile $N_{1}$ is shown in Fig. 6(b) (the larger the amplitude of $N_{1}$, the higher the lateral SHB). The laser is type A with $\kappa L=3.2$ and the effective diffusion length is set to $L_{\text {eff }} / w=1.6$. The laser is modulated with a step function.

At time $S$ when the first overshoot happens, lateral SHB occurs strongly at the phase-shifted region [in other words $N_{1}$ is large; see Fig. 6(b)] and the average gain in the phase-shifted region is reduced by the lateral SHB through the parameter $\xi_{1}$. The reduction of gain has the effect of increasing the average carrier concentration $N_{p}$ at the phase-sift region. This reduces the longitudinal SHB [see Fig. 6(c)]. Similar effects also occur for $\kappa L=0.8$ and 1.24. From the above it is clear that lateral effects on DFB lasers are different from those in FP lasers because the dynamic behavior of DFB lasers is strongly affected by their longitudinal uniformity.

The damping caused by lateral SHB and diffusion can be explained in terms of gain saturation [5], but only when $L_{\text {eff }} / w \geqslant 1$. For other values of $L_{\text {eff }} / w$ the present more complex analysis must be employed. Statically, the effect of carrier diffusion is straightforward: gain compression results from spatial hole burning, which increases with decreasing $L_{\text {eff }}$. Dynamically, although short carrier diffusion length reduces gain, it does not cause the damping associated with conventional gain compression. Under large-signal modulation, strong damping only occurs for the special case of $L_{\text {eff }} / w \approx 1$ and the oscillation strength is determined by the structure of the laser. The oscillation strength is also affected by the parameter $\xi_{1}$ given in (12). Fig. 7 shows that $\xi_{1}$ has a significant effect on damping (using the same laser parameters as in Fig. 6). From (12c), $\xi_{1}$ is strongly related to the rate of change of the lateral carrier profile $d N_{1} / d t$, which hence, has an effect on damping. This is an additional term to those employed in [5].

\section{B. Side Mode Suppression Ratio of the Longitudinal Modes}

As we have shown above, if the lateral SHB is strong in the phase-shifted region, it will tend to reduce longitudinal SHB. However, for lasers with large $\kappa L(>2.4)$ and with a relatively short lateral carrier diffusion length, strong lateral SHB can occur near the facets rather than the phase-shifted region. This will enhance longitudinal SHB and reduce SMSR.

Fig. 8 shows the dynamic response of a laser under large-signal modulation. The laser is type $\mathrm{G}$ with $\kappa L=2.6$ (i.e., $\Gamma_{y}=0.95, \xi_{1}=0.45$, and $\xi_{2}=0.3$ ). The normalized 


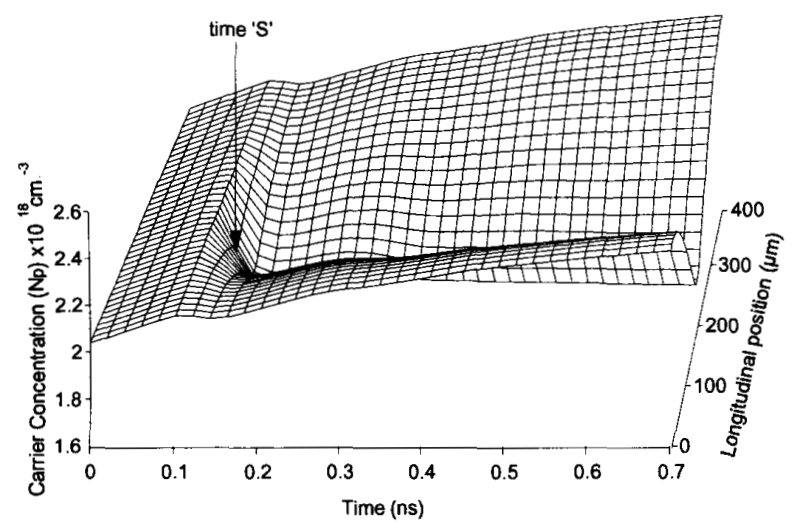

(a)

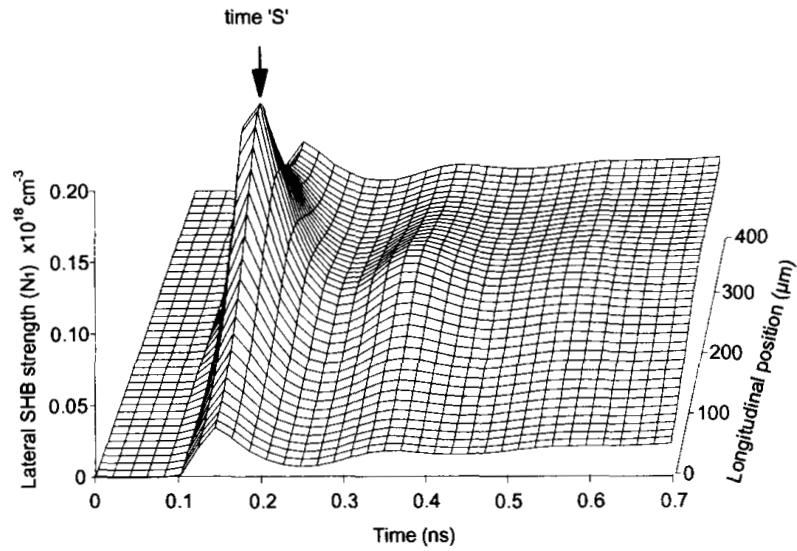

(b)

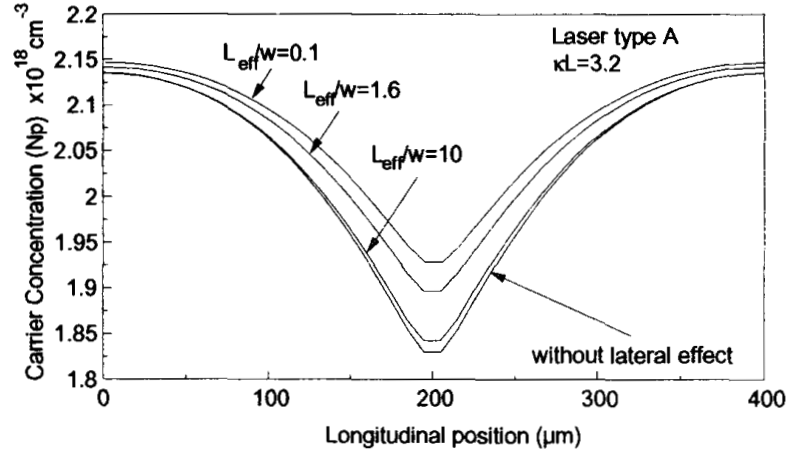

(c)

Fig. 6. (a) Large-signal modulation transient response of the cross-sectional average carrier concentration profile $N_{p}$. The laser has $k L=32$ with structure of type A. The normalized carrier diffusion length $L_{\text {eff }} / w$ is set to 1.6. (b) Large-signal modulation transient response of the latera SHB strength $\left(N_{1}\right)$ along the laser cavity. At time equal to $S$ when the first overshoot occurs, the lateral SHB strength is high in the center of the laser cavity. (c) Comparison of the longitudinal carrier distribution with and without lateral carrier diffusion at time $S$ when the first overshoot occurs. The normalized effective carrier diffusion length is set to $0.1,1.6$, and 10.0

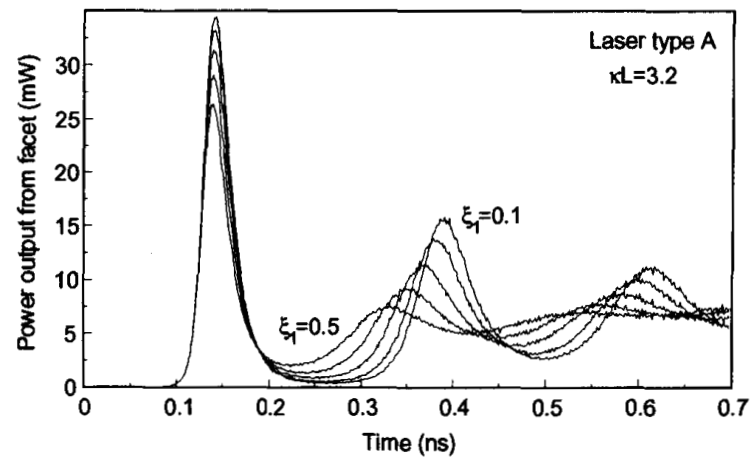

Fig. 7. Comparison between the transient response of power output from the facets with $\xi_{1}$ varied from 0.1 to 0.5 . The laser has $\kappa L=3.2$, is of type $\mathrm{A}$, and is under large-signal modulation. The normalized effective carrier diffusion length is set to $L_{\text {eff }} / w=1.6$.

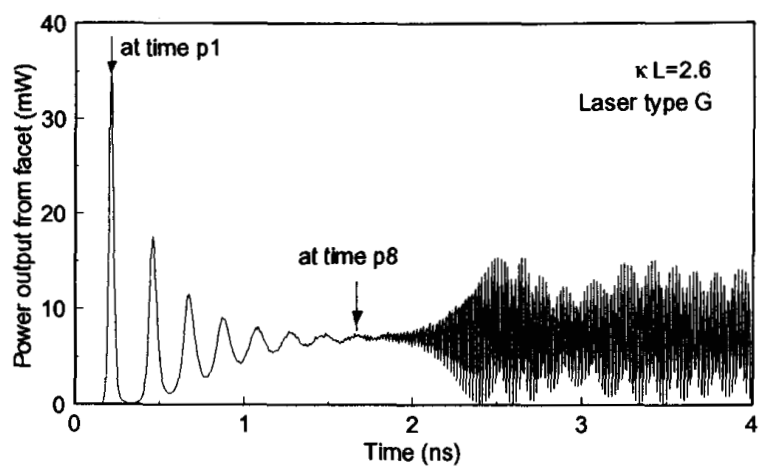

(a)

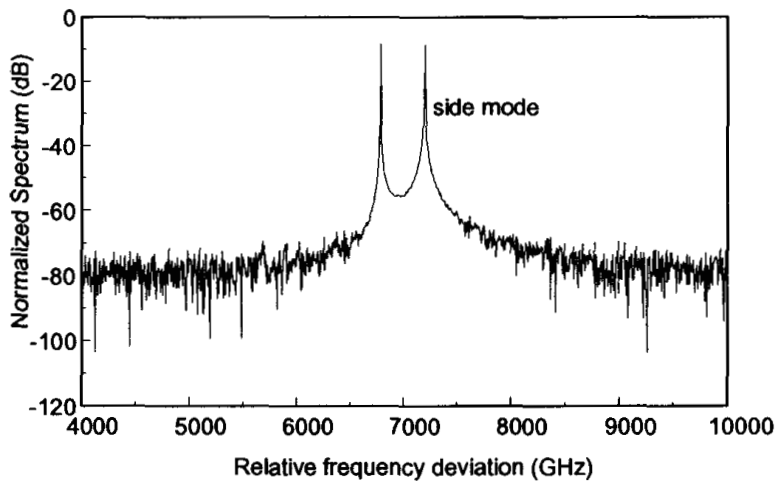

(b)

Fig. 8. (a) Large-signal modulation transient response of the output optical power. The laser is type $G$ with $\kappa L=2.6$. The normalized effective carrier diffusion length is set to $L_{\text {eff }} / w=0.1$. Mode beating starts at time $p 8$, caused by the side mode interfering with the main mode. (b) Fourier transform spectrum of time domain signal (last $1 \mathrm{~ns}$ ) given in (a).

lateral carrier diffusion length is set to $L_{\text {eff }} / w=0.1$. The laser is divided into 200 sections to ensure the accuracy of the calculation. The laser is first biased at threshold (around $2300 \mathrm{~A} / \mathrm{cm}^{2}$ ) and then switched to a level of 5600 $\mathrm{A} / \mathrm{cm}^{2}$. Fig. 8(a) shows the transient response of the laser. The laser switches on with a single-mode pulse (with 
no mode beating), but after nearly $2 \mathrm{~ns}$, the output power starts to oscillate as a consequence of the beating of two modes. The optical spectrum can be obtained by Fourier transform of the time-dependent field. Fig. 8(b) shows the spectrum of the oscillating signal taken from 3 to $4 \mathrm{~ns}$ in which two modes can be clearly seen. However the beating can be removed if we increase $L_{\text {eff }} / w$ to 2.0 or higher and this is strongly dependent on the structure of the lasers.

Dynamic changes to the side modes can be understood from the dynamic variation of the lateral SHB strength $N_{1}$ shown in Fig. 9(a). We can clearly see that the longitudinal profile of $N_{1}$ changes dramatically after the laser is switched on for 1 ns. The profile changes from convex to concave. This implies that strong lateral SHB is changed from around the phase-shifted region to near the end facets. Referring to Fig. 8(a), the average carrier profiles $N_{p}$, at time $p 1$ (the first overshoot) and at time $p 8$ (the eighth overshoot) are shown in Fig. 9(b) and (c) together with similar profiles where the lateral effect has been ignored. At time $p 1$, the longitudinal SHB is reduced by the lateral SHB as in Fig. 6(c). However, at time $p 8$, the longitudinal SHB is enhanced and reduces SMSR.

The final longitudinal carrier concentration profile (after a long time) is dependent on the strength of the side mode. Whatever the relative powers of the main and side modes, the carrier distribution will follow the total fieldintensity profile. Because the field-intensity profile of the side mode is more uniform than the main mode [16], the longitudinal carrier distribution profile has less SHB at the phase-shifted region when a side mode is present.

Fig. 9(d) shows the variation of the average SMSR with the normalized effective diffusion length for the laser parameters used earlier in this section. At $L_{\text {eff }} / w=0.5$, SMSR is about $10 \mathrm{~dB}$, reducing further for even lower values of $L_{\text {eff }} / w$. However, as $L_{\text {eff }} / W$ increases to 3 , SMSR goes up to around $50 \mathrm{~dB}$. This shows that high lateral SHB reduces SMSR.

We also investigate this effect in lasers with low $\kappa L(=$ 0.8 ) for the laser types $(A-G)$ shown in Table I. Fig. 10 shows the typical dynamic variation of lateral SHB strength $N_{1}$ of the lasers. The lateral SHB is strong near the facets and weak in the phase-shifted region. The shape of the lateral SHB strength does not have a significant change for $L_{\text {eff }} / w=0.1$. For low $\kappa L$ devices, single-mode operation remains undisturbed regardless of laser type.

\section{Effective Time-Averaged Linewidth Enhancement Factor}

The effective time-averaged linewidth enhancement factor $\alpha_{d}$ under large-signal modulation can be estimated from [17]

$$
\Delta f_{1 / 2} \Delta t_{1 / 2}=0.44 \sqrt{1+\alpha_{d}^{2}}
$$

where the time interval $\Delta t_{1 / 2}$ is the full-width half-maximum (FWHM) of the first overshoot pulse of the laser under large-signal modulation. $\Delta f_{1 / 2}$ is the FWHM of the spectrum of that pulse. This equation assumes that the first overshoot pulse has a Gaussian shape [17]. However,
non-Gaussian shapes can be accommodated by changing the coefficient 0.44 in (14) [20], although given the accuracy of the approximation, the shapes in this work can be regarded as Gaussian (see Fig. 5 and 6).

Fig. 11 shows the typical shape of the spectrum of the pulse. Because of the DFB spectrum "rabbit ear" [17] shape, it is difficult to define the FWHM width sensibly. In our calculation, the first spike is ignored and the spectral height for FWHM purposes is taken as that of the central part of the profile.

Fig. 12 shows the average variation of the normalized time-averaged linewidth enhancement factor with the normalized effective diffusion length. The calculation procedure is similar to that for the results shown in Fig. 3. The structures under investigation are $\gamma / 4$ lasers with $\kappa L=$ $0.8,1.24$, and 3.2. Only the laser structure types A and E are shown in the diagram.

In general, the time-averaged linewidth enhancement factor reduces with carrier diffusion length. This is due to the dynamic reduction of longitudinal SHB caused by lateral SHB [see Fig. 6(c)]. A maximum reduction of 55 percent of the time-averaged linewidth enhancement factor can be obtained. This reduction is strongly dependent on the structure of the laser. Lasers of type A have large reduction of the time-averaged linewidth enhancement factor as the lateral effect is enhanced.

\section{Discussion AND ConClusion}

From the analysis of Section III, the optimum width of the active region should be equal to or slightly less than (90 percent) the effective diffusion length of carriers in the InGaAsP active region. In addition, in order to obtain maximium coupling between the optical field and carriers in the lateral direction, the optical field should be completely confined in the active region. The effective carrier diffusion length in the active region is dependent on the mobility of the carriers and temperature and varies with external current injection. In FP InGaAsP lasers, the estimated value [2] is $2-3 \mu \mathrm{m}$, so the optimum width calculated from Section III can be realized in BH DFB lasers of this type.

The relaxation oscillation frequency $\omega_{r}$ and the damping frequency $\omega_{d}$ of DFB lasers vary with $\kappa L$ even if their optical power outputs are the same. The general expression of the relaxation oscillation frequency and the damping rate can be written as [18]

$$
\omega_{r}^{2}=\langle S\rangle g_{N} \nu_{g} / \tau_{p} \text { and } \omega_{d}=\nu_{g} g_{N} / \epsilon,
$$
respectively, where $\langle S\rangle$ is the average photon density within the active region and $\tau_{p}$ is the photon lifetime. Equation (15) is deduced from a simple photon rate equation and has already been applied to FP lasers. The equation can also be used to estimate the dynamic behavior of DFB lasers provided that we know their photon lifetimes and the photon density profiles.

Photon lifetimes of DFB lasers depend on the laser structure and the value of $\kappa L$. For symmetric $\lambda / 4$ phaseshifted DFB lasers with large $\kappa L(>3.0)$, photon lifetime is large compared to that for similar low $\kappa L(<1.0)$ 


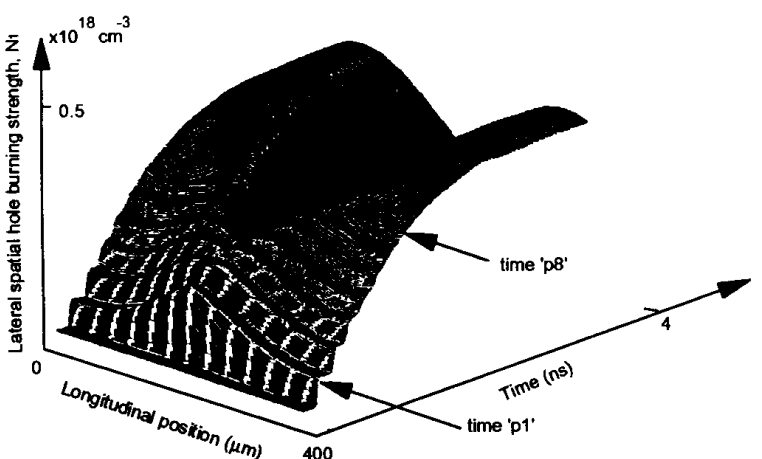

(a)

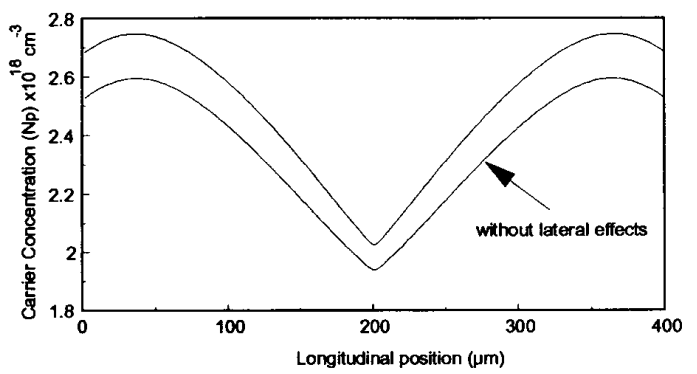

(c)

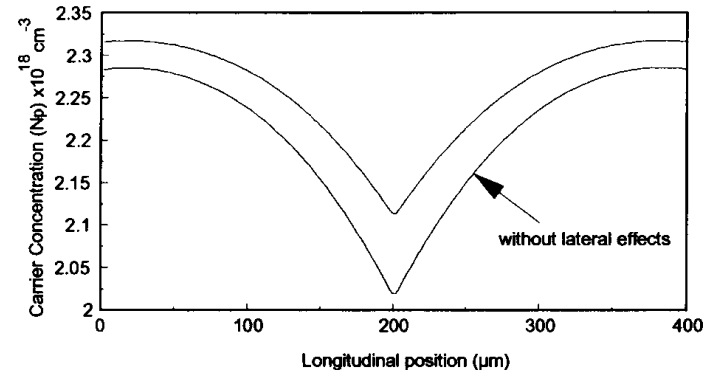

(b)

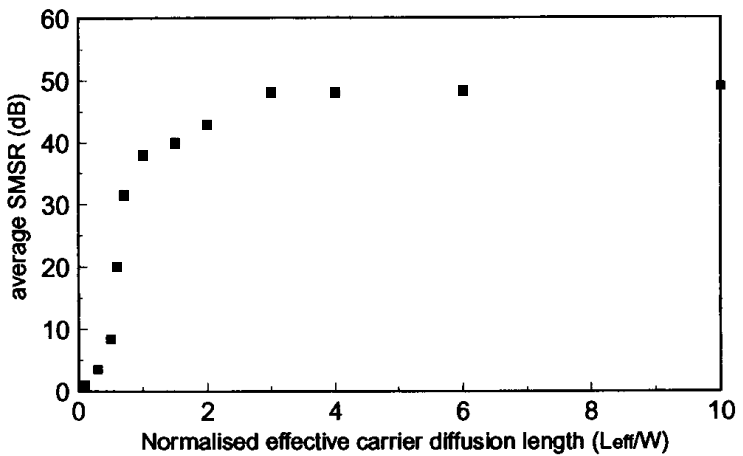

(d)

Fig. 9. (a) Large-signal modulation transient response of the lateral SHB strength $\left(N_{1}\right)$ along the laser cavity. At time $p 1$ when the first overshoot occurs, the lateral SHB strength is high in the phase-shifted region. At time equal to $p 8$ when the eighth overshoot occurs, the lateral SHB strength is high near the facets of the laser. (b) Comparison of the cross-sectional average carrier distributions $N_{p}$ with and without lateral carrier diffusion calculated at time $p 1$ when the first overshoot occurs [see Fig. 8(a)]. The difference in carrier concentration between the phase-shifted region and the facets is reduced by lateral carrier diffusion. (c) Comparison of the cross-sectional average carrier distributions $N_{p}$ with and without lateral carrier diffusion calculated at time $p 1$ when the first overshoot occurs [see Feg. 8(a)]. The difference in carrier concentration between the phase-shifted region and the facets is increased by lateral carrier diffusion. (d) Average side mode suppression ration (SMSR) against the normalized effective carrier diffusion length $\left(L_{\text {eff }} / W\right)$.

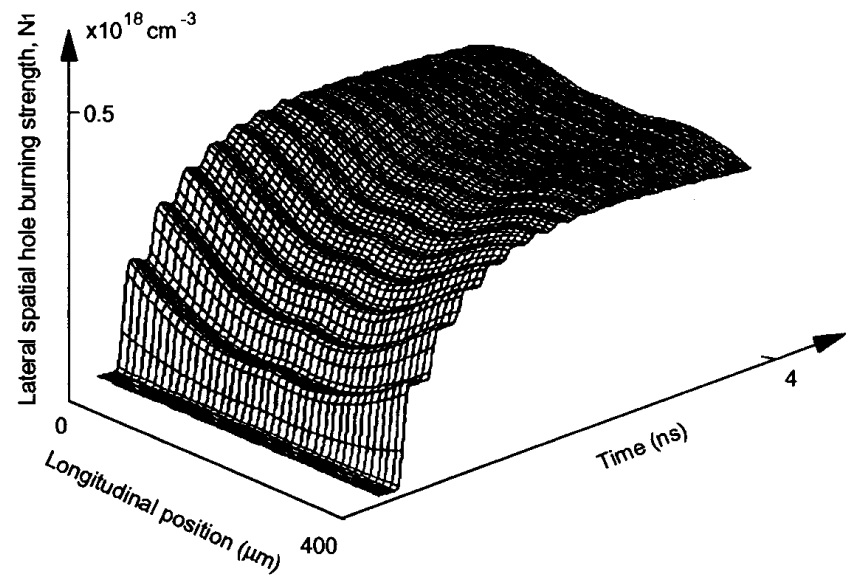

Fig. 10. Large-signal modulation transient response of the lateral SHB strength $\left(N_{1}\right)$ along the laser cavity. The laser has $\kappa L=0.8$ with structure of type G. The normalized carrier diffusion length $L_{\text {eff }} / w$ is set to 0.1 .

lasers. For high $\kappa L$, more photons accumulate in the phase-shifted region as evidenced by the low carrier densities near the center of the high $\kappa L$ laser in Fig. 4; this also implies that this structure has a relatively low loss $\left(\tau_{p}\right.$ is large). For low $\kappa L$, more photons are accumulated near the facets and relatively large losses are expected. However, applying the above to (14), this does not mean that low $\kappa L$ devices have relatively high resonance frequencies: this is because we have to take into account the total photon energy $\langle S\rangle$ stored inside the laser cavity. For 


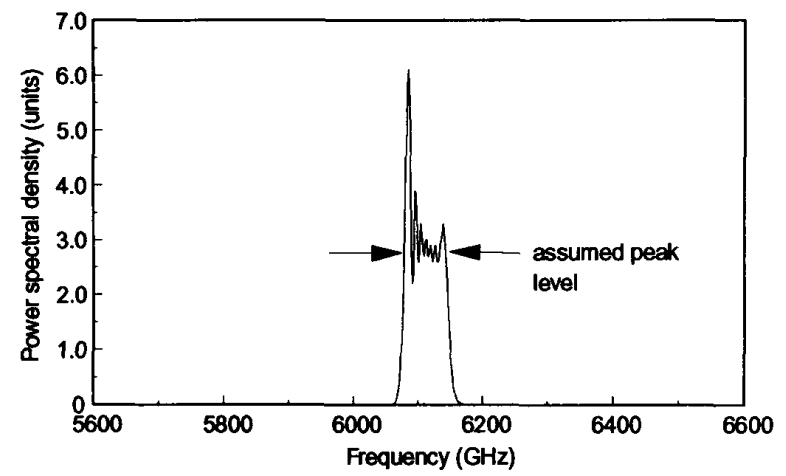

Fig. 11. Typical Fourier transform spectrum of the first overshoot pulse.

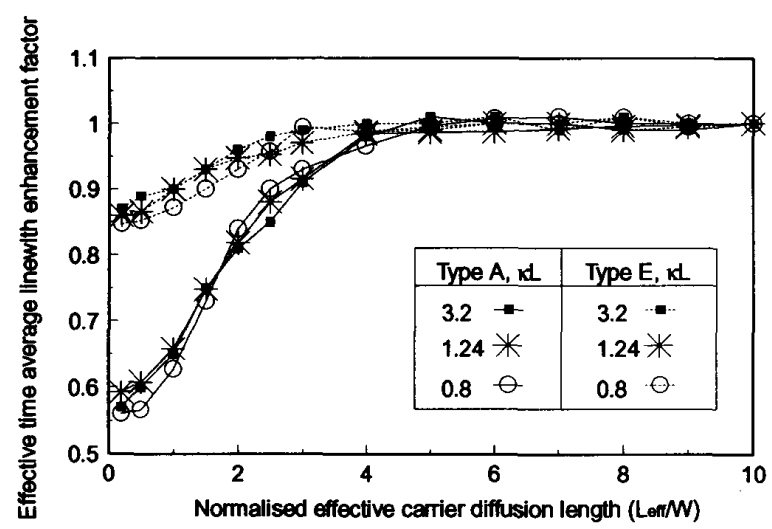

Fig. 12. Comparison of the normalized time-averaged linewidth enhancement factor of lasers with $\kappa L=0.8,1.24$, and 2.3 with the normalized effective carrier diffusion length varied from 0.2 to 10 .

lasers with same output power, the energy stored inside those with large $\kappa L$ is much larger than that in low $\kappa L$ devices. The resonance frequencies calculated in Section III are normalized; however, we should note that large $\kappa L$ devices have relatively large resonance frequencies, as shown above.

The damping frequency (or inverse damping rate) in (14) is independent of the structure of the laser and it is determined only by the gain compression factor $\epsilon$ and the differential gain. It is valid if the lasers have uniform longitudinal carrier profiles. In DFB lasers, $\epsilon$ is enhanced by longitudinal SHB [7]. For large $\kappa L$ devices, slight increases in damping rate are expected.

The lateral effect in symmetric $\lambda / 4$ phase-shifted DFB lasers with high $\kappa L(>3.0)$ causes increased relaxation oscillation frequencies and damping as well as reduced chirp. Large $\kappa L$ devices may also have a low threshold current density and a low linewidth-power product; see Whiteaway [16].

However, there is a major disadvantage of high $\kappa L$ devices, which is the low SMSR arising from longitudinal SHB. Although lateral SHB can reduce longitudinal SHB with a suitable choice of active region width, the optimum value still does not eliminate side modes for $\kappa L>3.0$. One of the possible ways to enhance the SMSR is to introduce first-order radiation loss $\left(h_{1}\right)$ [14]. We have performed extra calculations for the laser $(\kappa L=3.2)$ including a second-order grating. The same parameters were used as in Fig. 3, except $\alpha_{s}=15 \mathrm{~cm}^{-1}$ and $h_{1}=5.0$ $\mathrm{cm}^{-1}$. Similar results are obtained, showing $h_{1}$ does not degrade the performance of the devices, but the SMSR is enhanced by more than $30 \mathrm{~dB}$.

In conclusion, we have developed a detailed model to analyze the large-signal dynamic behavior of $\mathrm{BH}$ DFB lasers. By using the effective index method together with the perturbation method, the complex three-dimensional laser structure is reduced into a one-dimensional problem. We have taken into account lateral as well as longitudinal carrier variation and the consequent refractive index variation. To the best of our knowledge, this is the first time the large-signal two-dimensional dynamic behavior of a DFB laser has been considered.

This model has been used to analyze the large-signal dynamic behavior of symmetric $\lambda / 4$ phase-shifted $\mathrm{BH}$ DFB lasers. We found that lateral SHB and carrier diffusion have significant effects on the performance of such lasers. Damping rate, relaxation oscillation frequency, SMSR, and the time-averaged linewidth enhancement factor are affected. Laser structures with strong lateral optical field confinement and effective carrier diffusion length close to the width of the active region are optimum for relaxation oscillation frequency and damping, and the time-averaged linewidth enhancement factor is also reduced. The maximum reductions are more than 95 percent in relaxation oscillation strength and 30 percent in relaxation oscillation period. The effective timeaveraged linewidth enhancement factor under large-signal modulation can also be reduced by 40 percent.

We have demonstrated the importance of lateral effects on the dynamic behavior of DFB lasers. Ignoring the lateral effects may result in an inaccurate estimation or interpretation of the performance of DFB lasers. Although we only simulated symmetric $\mathrm{BH} \lambda / 4$ devices, other waveguide structures can also be modeled, as can lasers employing facet reflections or pure gain-coupled devices.

\section{APPENDIX I}

The transverse confinement factor $\Gamma_{x}$ is given by

$$
\Gamma_{x}=\int_{\substack{\text { active } \\ \text { layer }}} \phi_{0}^{2}(x) d x
$$

The longitudinal coupling coefficient $\kappa$ and the first-order radiation loss coefficient $h_{1}$ are given by

$$
\kappa=\frac{1}{2 \beta_{0}} \iint_{\substack{\text { grating } \\ \text { layer }}} k_{0}^{2} A_{-2}(x, y) \phi_{0}^{2}(x) \psi_{0}^{2}(y) d x d y
$$




$$
\begin{array}{r}
h_{1}=\frac{-i k_{0}^{2}}{2 \beta_{0}} \iint_{\substack{\text { grating } \\
\text { layer }}} A_{-1}(x, y) \phi_{0}(x) \phi_{-1}(x) \\
\cdot \psi_{0}(y) \psi_{-1}(y) d x d y
\end{array}
$$

where $A_{-2}$ and $A_{-1}$ are the Fourier coefficients of the dielectric grating and $\kappa_{0}$ is the wave vector in free space.

The lateral confinement factor $\Gamma_{y}$ is given by

$$
\Gamma_{y}=\int_{\substack{\text { active } \\ \text { layer }}} \psi_{0}^{2}(y) d y
$$

and the first-and second-order coupling parameters are given by

$$
\begin{aligned}
& \xi_{1}=\int_{-w / 2}^{w / 2} \cos (2 \pi y / w) \psi_{0}^{2}(y) d y \\
& \xi_{2}=\int_{-2 / 2}^{w / 2} \cos ^{2}(2 \pi y / w) \psi_{0}^{2}(y) d y .
\end{aligned}
$$

The fields given satisfy the normalization condition

$$
\int_{-\infty}^{\infty} \phi_{0}^{2}(x) d x=\int_{-\infty}^{\infty} \psi_{0}^{2}(y) d y=1
$$

and the transverse field can be solved using the same method as given in [19].

\section{REFERENCES}

[1] M. Ito, T. Ito, and T. Kimura, "Dynamic properties of semiconductor lasers," J. Appl. Phys., vol. 50, no. 10, pp. 6168-6174, 1979.

[2] K. Kishino, S. Aoki, and Y. Suematsu, "Wavelength variation of $1.6 \mu \mathrm{m}$ wavelength buried heterostructure GaInAsP/InP lasers due to direct modulation," IEEE J. Quantum Electron., vol. QE-18, pp. 343-351, 1982.

[3] W. W. Ng and E. A. Sovero, "An analytic model for the modulation response of buried heterostructure lasers," IEEE J. Quantum Electron., vol. QE-20, pp. 1008-1015, 1984.

[4] T. L. Koch and R. A. Linke, "Effect of nonlinear gain reduction on semiconductor laser wavelength chirp," Appl. Phys. Lett., vol 48 , no. 10 , pp. $613-615,1986$.

[5] R. Tucker and D. J. Pope, "Circuit modelling of diffusion on damping in a narrow-stripe semiconductor laser" IEEE J. Quantum Electron., vol. QE-19, pp. 1179-1183, 1983.

[6] N. Chinone, K. Alkl, M. Nakamura, and R. Ito, "Effect of lateral mode and carrier density profile on dynamic behaviors of semiconductor lasers," IEEE J. Quantum Electron., vol. QE-14, pp. 625-631, 1978.

[7] G. Morthier, F. Libbrecht, K. David, P. Vankwikelberge, and R. G. Baets, "Theoretical investigation of the second-order harmonic distortion in the AM response of $1.55 \mu \mathrm{m}$ F-P and DFB lasers," IEEE J. Quantum Electron., vol. 27, pp. 1990-2002, 1991.

[8] P. Vankwikelberge, F. Buytaert, A. Franchois, R. Baets, P. I Kuindersma, and C. W. Fredriksz, "Analysis of the carrier-induced FM response of DFB lasers: Theoretical and experimental case studies," IEEE J. Quantum Electron., vol. 25, pp. 2239-2254, 1989.
[9] M. J. Adams, An Introduction to Optical Waveguides. New York: Wiley, ch. 6, 1981.

[10] W. Streifer, D. R. Scifres, and R. D. Burnham, "Coupled wave analysis of DFB and DBR lasers," IEEE J. Quantum Electron., vol QE-13, pp. 134-141, 1977.

[11] M. Gault, P. A. Mawby, and M. S. Towers, "Solution to the wave equation in the numerical simulation of buried heterostructure lasers," IEE Proc. Pt. J, vol. 140, no. 1, pp. 44-48, 1993.

[12] L. M. Zhang and J. E. Carroll, "Large signal dynamic model of the DFB laser," IEEE J. Quantum Electron., vol. 28, pp. 604-611, 1992.

[13] A. J. Lowery, "New dynamic model for multimode chirp in DFB semiconductor lasers," IEE Proc. Pt. J, vol. 137, pp. 293-300, 1990.

[14] L. M. Zhang, S. F. Yu, M. C. Nowell, D. D. Marcenac, J. E. Carroll and R. G. S. Plumb, "Dynamic analysis of radiation and side mode suppression in second-order DFB lasers using time domain largesignal travelling wave model," IEEE J. Quantum Electron., 1994.

[15] D. D. Marcenac and J. E. Carroll, "A new quantum mechanical model for realistic Fabry-Perot lasers," IEE Proc. J, vol. 140, no. 3, pp. 157-171.

[16] J. E. A. Whiteaway, G. H. B. Thompson, A. J. Collar, and C. J. Armistead, "The design and assessment of $\lambda / 4$ phase-shifted DFB laser structures," IEEE J. Quantum Electron., vol. 25, pp. 1761$1779,1989$.

[17] A. J. Lowery, "Model for multimode picosecond dynamic laser chirp based on transmission line laser model," IEE Proc. Pt. J, vol. 135 , pp. $126-132,1988$

[18] K. Petermann, Laser Diode Modulation and Noise. Dordrecht, The Netherlands: Kluwer Academic, ch. 4, 1991.

[19] A. Hardy, D. F. Welch, and W. Streifer, "Analysis of second order gratings," IEEE J. Quantum Electron., vol. 25, pp. 2096-2105, 1989.

[20] J. E. Carroll, I. H. White, and D. F. G. Gallagher, "Dependence of chirp in injection lasers on temporal optical pulse shape," IEE Proc. Pt. J, vol. 133, no. 4, pp. 279-282, 1986.

S. F. Yu, photograph and biography not available at the time of publication.

R. G. S. Plumb, photograph and biography not available at the time of publication.

L. M Zhang, photograph and biography not available at the time of publication.

M. C. Nowell, photograph and biography not available at the time of publication.

J. E. Carroll, photograph and biography not available at the time of publication. 